\title{
POLÍTICAS DE POBLACIÓN EN LOS PAÍSES SOCIALISTAS DE EUROPA
}

\author{
Milos Maçura* \\ Instituto de Economía, Belgrado
}

A MEDIADos de los años sesenta, el surgimiento de políticas dirigidas a contrarrestar el descenso rápido de la fecundidad en algunos países socialistas de Europa, atrajo gran interés, así como especulación e investigación. El descenso de la fecundidad era ya notorio en el occidente y el sur de Europa a finales del período de entreguerra. Pero en la postguerra su descenso fue más pronunciado y el impacto de una tasa de reproducción por abajo de la unidad fue lo suficiente fuerte como para inducir políticas nunca pensadas en los años cincuenta. El estudio de la fecundidad se colocó, así, en el centro de atención de las políticas relacionadas con la población. Ni la migración internacional, ni la migración interna o la urbanización, así como tampoco la estructura de edades ni la mortalidad, recibieron atención parecida.

La situación era semejante a la de los países escandinavos en el decenio de 1930, si bien bajo diferentes condiciones económicas y sociales. Las respuestas tanto del público como del planificador fueron también semejantes, pero tuvieron lugar bajo un contexto político y social diferente. Existieron, pues, semejanzas y diferencias, las primeras tal vez con respecto a los objetivos sociales y demográficos y las segundas en cuanto a la amplitud de las medidas. Sin embargo, el análisis comparativo de políticas desarrolladas en los años treinta por los socialdemócratas escandinavos y en los sesenta por los socialistas marxistas no es tema de este trabajo. El paralelo, en la medida en que existió, fue mencionado incidentalmente, como eco de un remoto pensamiento acerca de la compleja relación entre el cambio de la población y la respuesta política.

Sería equivocado pensar que el desarrollo de políticas que afectan a la población en los países socialistas de Europa constituyó un proceso uniforme. Las actitudes gubernamentales y las políticas variaron en muchos aspectos, así como las medidas mismas; la duración y el énfasis fueron, asimismo, bastantes diferentes, Las políticas nacionales han sido discu-

* Agradezco al Dr. Bernard Berelson por animarme a escribir este trabajo y por la oportunidad de presentarlo en la reunión anual de la Asociación de Población de Norteamérica, en 1973. El artículo original ha sido revisado ligeramente para su publicación. La traducción del inglés fue hecha por Abelardo Hernández Millan. 
tidas en diversos artículos accesibles al público internacional. No es mi intención, por lo tanto, hacer un análisis comparativo de las políticas de población nacionales de los países socialistas de Europa.

El propósito de este trabajo es resumir los factores que han influído sobre la elaboración de políticas y señalar las principales tendencias de las políticas de población recientes.

\section{RESUMEN DE TENDENCIAS Y POLíticas}

De nueve países socialistas que existen actualmente en Europa, sólo dos no tuvieron, a principios de los años veinte, tasas de natalidad por encima de 30 por mil habitantes y tasas de mortalidad de más de 20 por mil. A finales de los sesenta, todos estos países, menos uno, habían alcanzado ya una avanzada etapa de la transición demográfica. La tasa de fecundidad en Bulgaria, Checoslovaquia, República Democrática Alemana (RDA), Hungría, Polonia, la URSS y Yugoslavia oscilaba entre 14 y 17 por mil, en Rumania era de 21 por mil y en Albania superior a 35 . La tasa de mortalidad en la RDA era de 14 por mil, en Checoslovaquia y Hungría se encontraba arriba de 11 , y en el resto de los países estaba entre 8 y 10 por mil. El proceso de envejecimiento de la población se desarrolló rápidamente en la RDA, ligeramente menos en Checoslovaquia y Hungría, y se anticipó en forma aún menos rápida en los otros países. La pirámide por sexo y edad se transformó seriamente por las pérdidas de guerra en la RDA, Polonia, la URSS y Yugoslavia. La emigración registrada durante los años cincuenta y sesenta fue impresionante en la RDA, más bien moderada en Yugoslavia, poco menos que moderada en Polonia y Rumania y poco apreciable en Bulgaria y Hungría. No hubo un sólo país socialista en Europa con una ganancia neta en población debida a la inmigración.

Como consecuencia del interjuego entre natalidad, mortalidad y migración, en Albania, la población fue 83\% mayor en 1970 que en 1950; $34 \%$ en la Unión Soviética, $30 \%$ en Polonia, $25 \%$ en Rumania y Yugoslavia, entre 10 y $17 \%$, en Hungría, Bulgaría y Checoslovaquia, mientras que en la RDA fue $9 \%$ menor. La tasa anual media de crecimiento de la población entre 1950 y 1970 , fue de $3.1 \%$ en Albania, $1.5 \%$ en la Unión Soviética, $1.4 \%$ en Polonia, $1.1 \%$ en Rumania y Yugoslavia, $0.9 \%$ en Bulgaria y Checoslovaquia, $0.5 \%$ en Hungría y $-0.5 \%$ en la RDA. La tasa de crecimiento de la población urbana fue, en promedio, menor a $2 \%$ anual en la RDA, Checoslovaquia y Hungría, y mayor a $3 \%$ en los demás países. No fue sino hasta 1970 que la RDA logró una notable urbanización ( $81 \%$ de población urbana); La Unión Soviética, Polonia, Checoslovaquia y Bulgaria se urbanizaron a medias alcanzando entre 56 y $51 \%$ de población urbana, mientras que los demás países permanecía predominantemente rurales (entre 43 y $37 \%$ de población urbana).

El proceso de desarrollo, así como las políticas económicas y sociales pertinentes, son guiadas y coordinadas en todos estos países bajo consi- 
deraciones de planificación de mediano alcance. Sin embargo, hay marcadas variaciones con respecto al radio de acción, los niveles y la "orientación" de la planificación. Durante los últimos diez años o más, los aspectos de la población han recibido un lugar más amplio en la planificación del desarrollo que anteriormente, principalmente sobre la base de consideraciones económicas y sociales. Lo que es más, recientemente algunos países han dado nuevo énfasis a la población, considerando también los beneficios demográficos de un cambio deseado en las tendencias de la población.

En lo que toca a la fecundidad, en cinco países las políticas han sido encauzadas explícitamente hacia una mayor reproducción que la actual: Bulgaria, Checoslovaquia, RDA, Hungría y Rumania. ${ }^{1}$ De los otros países, tres han experimentado, en los años sesenta, tanto una tasa bruta como una tasa neta de reproducción menor que la unidad; Checoslovaquia tiene una tasa neta para todo el país por debajo de la unidad, pero en la región Checa, tanto la tasa neta de reproducción como la bruta, están por debajo de ese nivel; en la RDA ambas tasas están por arriba de 1 , pero con una tasa de crecimiento de la población negativa debida a la emigración.

Sin hacer especial referencia a objetivos demográficos, Yugolavia ha adoptado la planificación familiar como una política nacional, principalmente sobre la base de considerar los derechos individuales y humanos. ${ }^{2}$ En la URSS, el debate sobre las interrogantes de la población continúa todavía, principalmente entre demógrafos y economistas. Muchos de ellos, como Valentei, consideran que hay una "urgente necesidad de activar una política demográfica..." que debe ser conducida "por el Partido y el Estado, con la ayuda de medidas legislativas, administrativas y económicas." 3 En Polonia, después de algunos cambios de actitud del gobierno con respecto a la política de fecundidad, ha habido también una gran discusión acerca de la política a seguir. ${ }^{4}$ En Albania según algunas fuentes, no existe debate alguno acerca de políticas de población ni en los círculos políticos ni en los técnicos.

La política que suscita menos controversia, la que trata de la reducción de la mortalidad, se encuentra implícita en las políticas sociales y de salud de los nueve países en cuestión. La seguridad social compulsiva para todos o algunos grupos de la población, como puede ser el caso en países individuales, así como el desarrollo de los servicios de salud y de sus facilidades, ha conducido, claramente, al mejoramiento de la salud de la nación. Esto implica una disminución de la mortalidad, aunque no se

1 Jerzy Berent, "Causes of Fertility Decline in Eastern Europe and in Soviet Union”, “Population Studies, V. XXIV, Núm. 2, 1970, pp. 286-290.

2 Decisión acerca de la planificación familiar adoptada por la Asamblea Federal el 25 de Abril de 1969.

3 D. I. Valentei, "Acerca del comportamiento demográfico: problemas de la vida social", traducido de Pravda, 8 de enero de 1973; V. S. Stashenko y V. P. Piskunov, "Problema narodnonaselenia" (El problema de la población), Demograficheskie Tetradi, Núm. 8, Kiev, 1973. pp. 23-42.

4 Janusz Ziolkovski, véase la nota 25. 
hayan establecido objetivos específicos en relación a la reducción de la mortalidad ni a sus aspectos específicos. Sin embargo, en aquellos países que tienen una política que favorece la fecundidad, la baja mortalidad infantil y la protección y cuidado de los niños están relacionadas, de una $\mathrm{u}$ otra forma, el patrón deseado de fecundidad. Si esta observación es correcta, significa que minimizar las defunciones infantiles es considerado como algo asociado estrechamente con la optimización de los nacimientos, lo cual constituye de hecho, lo que se ha denominado "reproducción racional y humana".

Con excepción de las proyecciones demográficas, no se ha dado un lugar prominente al criterio y a los objetivos relacionados con el crecimiento y el tamaño de la población. Los planes de desarrollo y los documentos de políticas, no parecen contemplar objetivos específicos con respecto al tamaño de la población que debe ser alcanzado en el futuro. Sin embargo, la estructura de edades de la población ha recibido gran atención, particularmente en relación con las estimaciones de mediano y largo alcance de la oferta futura de mano de obra. Parece que el cambio esperado en la composición de edades ha sido un factor de importancia y, consecuentemente, una inspiración para las políticas desarrolladas en los años sesenta. ${ }^{5}$

La redistribución de la población dentro del territorio nacional y el crecimiento de la población urbana, basada principalmente en la migración desde las áreas rurales a las áreas urbanas es considerada, al menos en seis naciones, como una componente importante del desarrollo regional. El cambio de fronteras nacionales en la postguerra y los movimientos internos y externos relacionados a este cambio, fueron seguidos por una migración auspiciada por el Estado y por programas de colonización por lo menos en cuatro países. Pero estas fueron políticas momentáneas diseñadas e implementadas para responder a las condiciones de postguerra. La migración trascontinental hacia las regiones orientales y el desarrollo de nuevos pueblos y ciudades pareció ser una política de largo plazo en la URSs, mientras una variedad de políticas relativas a la migración interregional y rural-urbana parecían prevalecer en países como Polonia, Checoslovaquia, Rumania y Yugoslavia. ${ }^{6}$

Las políticas relativas a la migración internacional, aunque no fueron establecidas explícitamente, aparecieron en todos los países, menos en Yugoslavia, con miras a desalentar los movimientos internacionales. Una migración temporal reducida de trabajadores, que involucró casi a seis países, fue organizada sobre bases bilaterales en los años sesenta. Bulgaria, Polonia y Hungría aparecieron como países de origen y la RDA, Checoslovaquia y la Unión Soviética como países receptores. En un estudio

5. Dr. Timar Yanosh, Polozhenie zaniatosti v stranah - članah SEV s 1950 po $1990 \mathrm{gg}$. (Situación del empleo en los países del Consejo de Ayuda Mutua Económica, 1950-1990), Budapest, noviembre, 1972, 200 pp. y Anexo.

6 Kosta Mihailovic, Regional Development: Experiences and Prospects in Eastern Europe, Mouton, 1972, 225 pp.

7 Minko Minkov. Migracia na naselenito, Partizdat. Sofía, 1972, 207 pp. 
de la migración búlgara, Minkov consideraba poco satisfactoria la migración internacional y aboga en favor de la migración internacional temporal de trabajadores dentro de los países socialistas miembros del Consejo de Ayuda Mutua Económica. ${ }^{7}$ El tema parecía atractivo, pero todavía se encuentra en manos de los técnicos. En Yugoslavia se ha desarrollado, desde mediados de los años sesenta, una política de permisos con respecto a una migración temporal, seguida, sin embargo, con gran preocupación. Los acuerdos bilaterales con seis gobiernos de los países europeos occidentales reflejan la posición política respecto a las cuotas y a los derechos individuales y sociales de los trabajadores empleados en el exterior. Algunos acuerdos relativos a esquemas de trabajo intensivo, como el de la construcción, han sido hechos también por algunos países socialistas. Por lo que toca a Albania, no existe ninguna política relativa a la migración, ni tampoco la migración internacional se está llevando a cabo de forma que afecte las tendencias de la población.

\section{IDEOLOGÍA Y TEORÍA}

En esta etapa pueden ser pertinentes algunas observaciones acerca de las cuestiones teórica e ideológicas relevantes para la política de población. Difícilmente puede decirse que, dentro del pensamiento marxista, haya sido mencionada alguna cuestión con respecto a la mortalidad y el cambio en la estructura de edades. El estudio de Roset acerca de la edad de la población puede apoyar esta afirmación. ${ }^{8}$ Por lo que toca al crecimiento de la población, entre la mayoría de los escritores marxistas prevalece un optimismo basado, en apariencia, en consideraciones relacionadas a una baja densidad de población, y a la creencia en el progreso humano en general y en el de la ciencia y la tecnología en particular. Esta actitud es también básica en los debates acerca de la fecundidad y de su control, aunque esta cuestión es más compleja, como veremos más adelante. La interpretación marxista de las estructuras dinámicas tiene implicaciones amplias en el estudio del cambio de la población y de la fuerza de trabajo. En el presente contexto, es relevante a la redistribución de la población y al desarrollo urbano. Finalmente, el concepto de internacionalismo y solidaridad proletaria pueden ser considerados como la plataforma ideológica de la teoría de migración internacional, aunque esta última todavía no se haya desarrollado y su relación con la primera sea aún remota y oscura.

Casi no hay necesidad de comentar las proposiciones marxistas básicas relativas a la población y a la sociedad. La crítica del principio malthusiano y de "la ley universal de población"; la afirmación de que cada tipo de sociedad tiene su "ley peculiar de población" propia, la cual es "históricamente determinada dentro de sus propios límites"; la teoría de la plusvalía y de la superpoblación relativa; el papel dual de la po-

8 Edward Rosset, Proces starsenia sie ludnosci (El proceso de envejecimiento de la población), Versovia, 1959, $753 \mathrm{pp}$. 
blación como consumidor y productor; y la apreciación de una "posibilidad abstracta" de sobrepoblación que podría exigir de una sociedad comunista que "regule la producción de seres humanos", son bien conocidos de la mayoría de los lectores. ${ }^{9}$ Lo que interesa aquí es seleccionar los postulados teóricos e ideológicos demográficamente relevantes, para poder especular acerca de la manera en que éstos pueden influir sobre las políticas de población en una sociedad socialista. No es necesario decir que la "determinación histórica" de esos postulados implica que las políticas están influida por la situación demográfica y sus correlatos económicos y sociales.

El optimismo socialista tiende a generar políticas en las que se propugnan niveles altos, tanto en la fecundidad como en el crecimiento de la población. El argumento es simple y persuasivo: si la organización social y no la población, es la responsable del dilema malthusiano, una reorganización de la sociedad conduce, por sí misma a la solución del problema. Al contrario de lo que sucede en el capitalismo, la sociedad socialista es capaz de superar la superpoblación relativa y de absorber un crecimiento rápido de la población. Esta proposición, generalmente atribuída a Smulevich, ${ }^{10}$ fue debatida por Strumilin, quien sugirió que las leyes socialistas no eran las leyes capitalistas puestas al revés, sino que el asunto era más complejo, particularmente en lo que se refería a la relación inversa entre el ingreso y las tasas vitales. ${ }^{11}$

Asimismo, algunas otras consideraciones tanto teóricas como ideológicas han contribuido a la complejidad del tema. En una sociedad socialista, la supresión de la relación inversa entre tamaño de familia y niveles de salarios, hecho que había sido señalado por Marx como un subproducto del capitalismo, ${ }^{12}$ tiende a reducir la mortalidad y el número de hijos nacidos en una familia. La igualdad de oportunidades para ambos sexos y el reconocimiento de un estatus superior de la mujer, propios de una sociedad socialista, conducen hacía un crecimiento moderado de la población, como ya observaba Bebel. ${ }^{13} \mathrm{El}$ progreso económico y los

9 Un resumen de la tería marxista aparece en Naciones Unidas, Los determinantes y las consecuencias de las tendencias de la población, Segunda edición, Capítulo III.

$10 \mathrm{~B}$. Smulevich, Burzhnoaznye teorii narodonaselenia $v$ svete marksiskoleninskoy kritiki (Las teorías burguesas de la población a la luz de la crítica marxista-leninista), Moscú, 1936.

11 S. G. Strumilin, "K probleme razhdaemosti v rabochem srede" (Acerca de la fecundidad de la clase obrera), Problemi economiki truda (Problemas de economía del trabajo), Gosplanizdat, 1957.

12 Refiriéndose a la reproducción del grupo "sobrante" de la superpoblación, Marx observaba que este era "un elemento de la clase obrera que se reproduce asímismo y se eterniza, entrando en una proporción relativamente mayor que los demás elementos en el crecimiento total de aquella. De hecho, no sólo la masa de los nacimientos y defunciones, sino también la magnitud numérica de las familias se halla en razón inversa a la cuantía del salario, es decir, de la masa de medios de vida de que disponen las diversas categorías de obreros, El capital, Vol. I, México, Fondo de Cultura Económica, 1959, p. 545.

13 Bebel, Die Frau und Sozialismus, (1894). 
cambios asociados de la población, desde las industrias agrícolas a las no agrícolas, y desde las áreas rurales a la urbanas, proclamados por Marx, Lenin y otros marxistas, tienden también a reducir la fecundidad. ${ }^{14}$ La legalización del aborto, que probablemente fue un resultado del comentario hecho por Lenin acerca de la "libertad para la propaganda médica y la protección de los derechos democráticos de los ciudadanos", y que no tenía nada que ver con la mentalidad malthusiana, ${ }^{15}$ favorece también el descenso de la fecundidad. Debería mencionarse que la mayoría de los juicios de valor y las normas sociales mencionadas antes, raramente eran cuestionadas en un debate sobre políticas de población. La política de población dirigida a la fecundidad, tenía así que operar en un marco mucho más complejo, el cual se complicaba todavía más por las aspiraciones y privaciones de una sociedad en crecimiento.

Recientemente se han adelantado nuevos argumentos en favor de una política de fecundidad más alta en sociedades socialistas de baja fecundidad. La mayoría de estos argumentos no eran de naturaleza teórica o ideológica, sino que se derivaban del análisis y de las proyecciones disponibles para ciertos momentos dados.

Lucan, el Viceprimer ministro de Checoslovaquia, argumentaba que "el objeto principal del desarrollo demográfico", en una situación de baja fecundidad, "es el tamaño de las nuevas generaciones". Algunos economistas y planificadores de la fuerza de trabajo como Timur, abogan por una fecundidad más alta para contrarrestar un próximo descenso de la fuerza de trabajo. El envejecimiento de la población y "el deterioro de la composición por edades" fue también considerado como razón para una fecundidad más alta. Es de notarse que en los desarrollos recientes, los nuevos argumentos son de mucho más peso que el mero optimismo general, aunque el papel de éste último no pueda negarse.

La política relativa a la migración, redistribución regional de la población y urbanización es vista por algunos marxistas como parte de una política de población más amplia, mientras que otros la consideran como una componente del desarrollo regional. La teoría marxista del desarrollo regional tratada primeramente por Engels, ${ }^{16}$ y suplementada por Lenin ${ }^{17}$ fue bastante adecuada a una política de urbanización para aquellos países predominantemente rurales. Esta teoría, en principio era consistente con un antiguo objetivo del socialismo, el de la "supresión de la contradicción entre la ciudad y el campo". Pero el objetivo permaneció solamente como un asunto ideológico, dado que las políticas de desarrollo usualmente ponían mayor énfasis en el desarrollo urbano en tanto proceso gemelo al de industrialización.

14 En El desarrollo del capitalismo en Rusia (1899), Lenin hace un análisis concienzudo de los cambios de población de las industrias agrícolas a las no agrícolas, así como del crecimiento urbano. Sin embargo, no hace referencia al hecho de que este cambio conducía a la disminución de la fecundidad.

15 V. I. Lenin, "Rabochy Klass i neomaltuzianstvo (1913).

16 Friedrich Engels, Die Lage der Arbeitenden Klasse in England (1845).

17 V. I. Lenin, El desarrollo del capitalismo en Rusia (1899). 
Los conceptos socialistas de igualdad y solidaridad, junto con las consideraciones relativas a la distribución regional de recursos fundamentales fueron básicos para la aparición de otra dimensión, constituida por el desarrollo regional. La teoría inicial enfocaba la distribución de las "fuerzas productivas", o sea, de la asignación, por medio de una planificación central, tanto de medios de producción como de fuerza de trabajo, hacia regiones particulares. ${ }^{18} \mathrm{La}$ migración fue concebida como un proceso planeado cuyo objetivo principal era el de balancear los requerimientos regionales de fuerza de trabajo.

Se ha registrado un progreso significativo en la teoría del desarrollo regional durante los últimos dos decenios o más. Después de haber reconsiderado la teoría de los polos de desarrollo de Perroux, Mihailovíc sugirió que de acuerdo a la interpretación marxista de la dinámica de las estructuras, tanto los factores económicos y demográficos así como los sociales, son activados en el proceso de desarrollo regional. La migración de fuerza de trabajo y de población, especialmente la migración hacia las ciudades, juegan en este proceso un papel importante. Concierne a las políticas de desarrollo regional movilizar todos los recursos necesarios, e impulsar la distribución de población que concordará con el progreso tanto regional como por rama de producción. Las implicaciones programáticas de la teoría del desarrollo regional con respecto a los movimientos de población parecen obvias. Aún así, permanecen sin considerar cierto número de interrogantes, especialmente si la urbanización y la migración son vistas en relación con otros aspectos del cambio de la población.

Es difícil decir cuál pueda ser la consideración relativa a las políticas de migración internacional de los países socialistas europeos. No existe mucha literatura sobre el tema y sólo podemos especular acerca de las razones del tipo de políticas de emigración o inmigración en todos los países socialistas, a excepción de Yugoslavia. ${ }^{19}$ Conceptos ideológicos tales como el internacionalismo y la solidaridad proletaria, así como

18 K. Mihailović, op. cit.

19 O. T. Bogomolov, en su libro Teoria $i$ metodologia mezhdunaravnavo socialističeskavo razdelenia truda (Teoría y métodos de la división internacional socialista del trabajo), Moscú, 1967, 263 pp., analiza los diversos aspectos de la división del trabajo en la sociedad socialista, pero no hace mención de la migración internacional. M. Maçura y $T$. Mulina consideran la abundante mano de obra en Yugoslavia como una ventaja comparativa, y examinan una variedad de métodos relativos al empleo productivo, a los movimientos de capital e, inclusive, a la migración, "Perspektive korišcenja radnih potencijala Jugoslavije" (Consideraciones acerca de la futura utilización de la mano de obra potencial en $\mathrm{Yu}$ goslavia), Ekonomska Misao, Núm. 1, 1973, pp. 94-105. Rudolf Bakich, al considerar las necesidades futuras de fuerza de trabajo en Checoslovaquia hasta 1990, afirma" que es posible una colaboración en el campo de la fuerza de trabajo... con aquellos paises con reservas temporales de trabajo, con quienes se podría acordar una cooperación con base en los principios de la colaboración socialista" ("Problemas de recursos de mano de obra desde el punto de vista del desarrollo de la economía nacional de Checoslovaquia hasta 1990"); en Checo, mimeografiado. 
la teoría que ve en el socialismo un sistema mundial, hablan en favor de la migración internacional, al menos entre los países socialistas. Por otro lado, debe haber consideraciones políticas, o de otro tipo, que reglamenten la migración internacional como una proposición viable.

Un argumento económico en contra de la migración internacional puede buscarse en la naturaleza misma de la sociedad socialista, la cual es capaz de proporcionar empleo a todos aquellos que lo requieran. Consecuentemente, no hay necesidad de migración para equilibrar la oferta y la demanda de trabajo. En efecto, durante el período de planeación administrativa en todos los estados socialistas existió una situación de pleno empleo. Pero, en cuanto fue abandonada la rigidez administrativa, el subempleo vino a manifestarse y tuvieron lugar discusiones acerca del modelo "extensivo" versus el "intensivo" de la economía. La discusión de Minkov acerca de la migración internacional es tal vez un resultado de esos cambios. ${ }^{20}$

\section{LAS POLÍticAS DE POBLACIÓN Y LOS CAMBIOS DEMOGRÁFICOS}

De entre las distintas cuestiones discutidas en la literatura reciente, estamos interesados en una muy específica: la de si las políticas de los estados socialistas han sido desarrolladas ex ante, como una anticipación al cambio de población, o ex post, en respuesta a un cambio de población. La mayoría de las políticas económicas y sociales en estos estados son políticas ex ante y han sido formuladas en el proceso de planificación. Resulta apropiado, por tanto, examinar las políticas de población desde este ángulo particular.

No parece haber muchas políticas de población ex ante en los países socialistas europeos. Si hacemos a un lado las políticas de mortalidad y de salud, es la redistribución de la población la que se verá mayormente afectada por las políticas formuladas dentro de planes específicos. La migración hacía el oriente y el desarrollo de nuevas ciudades en la URSs, la redistribución regional de la población en algunos países, especialmente en Polonia, y las políticas en favor o en contra de la urbanización, tanto en los estados individuales como en períodos particulares, parecen estar bien coordinadas con las políticas económicas. Las políticas en contra de la emigración en todos los estados, menos en uno, pueden también considerarse como propias de este grupo de países.

Lo típico de tales políticas es que consisten regularmente de objetivos definidos, y en una diversidad de medidas que abarcan tanto incentivos económicos directos como indirectos y confianza en los factores de rechazo y atracción y en las medidas administrativas.

En cuanto a las políticas de fecundidad se refiere, la situación es ambigua debido a la complejidad y cronología para fijar los objetivos y adoptar medidas; debido a la combinación de medidas que probablemente

20 Minkov, op. cit. 
producen efectos pronatalistas o antinatalistas; y en virtud del hecho de que pueden adoptarse medidas en anticipación de efectos tanto demográficos como sociales, o de ambos. Aún más, ninguna política de población está aislada de consideraciones humanitarias, ideológicas, económicas, sociales, de salud y otras que son básicas a la sociedad. Esto es cierto particularmente para una sociedad socialista en la cual los problemas de población son considerados primordialmente como problemas pertenecientes a un contexto socioeconómico amplio.

Históricamente hablando, entre las muchas reformas y medidas llevadas a cabo en los días tempranos de los países socialistas, hubo algunas que por su misma naturaleza se esperaba que produjeran efectos demográficos. Pero no creo que estas medidas hayan sido promovidas a nombre de objetivos demográficos. Toda la reorganización de la sociedad fue encauzada hacía la afirmación de la clase trabajadora, la justicia social, la igualdad de oportunidades y la responsabilidad de la sociedad para el bienestar de grupos vulnerables. La ideología marxista fue la inspiración de la reorganización, ya que en ella misma se definen los objetivos sociales y económicos. Fueron también implementadas las medidas demográficamente relevante para dar vigencia a la teoría y a la ideología marxista. Hemos visto antes que, por lo que se refería a la fecundidad, esta ideología probablemente estimularía y, al mismo tiempo desestimularía los nacimientos. En las circunstancias dadas en todos los países socialistas, a excepción de uno, sucedió que la combinación de políticas y medidas fueron tales, que la transición demográfica sufrió una aceleración. También ocurrió que la disminución de la fecundidad descendió a un ritmo mayor que la mortalidad.

Esto ocurrió a pesar de que se esperaba, al menos en algunos países, que el crecimiento de la población permanecería en niveles altos. No sabemos mucho de las políticas antes de que estas fueran declaradas; la información adecuada está disponible a partir de mediados de los años cincuenta. Pero existieron antes, al menos en algunos países, enunciados de políticas ocasionales en favor de un crecimiento rápido de la población y de cifras grandes de población. Las teorías también han avanzado respecto a aquellas en la que se hablaba de una ley específica de población para el socialismo, manifestada en un crecimiento de población permanentemente rápido. El pleno empleo y las medidas económicas y sociales promovidas por el estado socialista, se creía, debían de resultar en una expansión demográfica continua. Ahora, post factum, nos damos cuenta que la combinación de medidas promovidas, no condujo hacia tal expansión y que dentro del sistema total de políticas y medidas, aquellas que tendían a reducir la fecundidad tuvieron mayor peso que las que se esperaba que lo mantuvieran alto.

Es tal vez debido a estos hechos que las políticas se han reconsiderado y rediseñado, desde mediados de los años sesenta, con el propósito de cotrarrestar aquellos factores y medidas que originaron que la tasa de reproducción se situara por debajo de la unidad. La política fue cambiada y nuevas medidas fueron expedidas en Rumania (1966), Bul- 
garia (en 1967 y 1968 y, nuevamente, en 1973), Hungría (1966-1968 y 1973) y Checoslovaquia (1968-1971). Las medidas que fueron adoptadas serán resumidas en la sección siguiente. En esta parte, solamente mencionaremos algunos enunciados de políticas hechas a propósito de algunas reformas.

El Consejo de Estado de Rumania, en su decreto de 1966, que prohibía el aborto inducido, aclaró que estas medidas eran adoptadas con base en consideraciones demográficas y de salud. Se consideró el aborto en "detrimento serio de la tasa de fecundidad y del incremento natural de la población". El Comité Central del Partido Comunista y el Consejo de Ministros de Bulgaria establecieron en 1967 que la nueva política dirigida a mejorar las condiciones, contribuía a impulsar la fecundidad y, consecuentemente, a un incremento en la tasa de crecimiento de la población. El primer Ministro de Checoslovaquia declaró en 1971 que su país estaba entre aquellos que tenían las tasas más bajas de fecundidad de la Europa de postguerra. "Consecuentemente, en el curso del quinto plan quinquenal, proporcionaremos apoyo social a las familias con hijos... Esperamos que las primas familiares aumente gradualmente, en particular en beneficio del segundo y del tercer hijo".

Parece, en consecuencia, que una nueva oleada de medidas de población ha sido lanzada en los estados socialistas como respuesta a la rápida disminución de la fecundidad.

Sin embargo, esto produjo también un efecto duradero ya que los gobiernos respectivos han decidido tratar los problemas de población sobre bases permanentes y de largo alcance. Se han establecido comisiones gubernamentales y comités de población y se ha decidido que las políticas de población sean incorporadas dentro de programas de desarrollo de mediano y largo alcance.

En cuanto a la República Democrática Alemana se refiere, se observa que no ha habido cambio en la política de población que fue adoptada a principios de los años cincuenta. Medidas restrictivas y positivas para impulsar la fecundidad han sido expedidas desde hace tiempo con objeto de mantener la tasa de incremento natural a un nivel satisfactorio. No hay especial necesidad en este país para innovar políticas relativas a urbanización, dado que el nivel de desarrollo urbano era ya de por sí elevado en los años cincuenta.

En Yugoslavia, hubo, desde principio de los cincuenta y dentro de los planes de desarrollo una política que impulsó los cambios de población desde las industrias agrícolas a las no agrícolas. La planificación familiar, como política nacional, fue proclamada en 1969 por la Asamble Federal. Esto fue hecho no en virtud de objetivos demográficos, sino sobre la base de consideraciones humanitarias, en las cuales, tanto el estatus como la salud de la mujer y las perspectivas para las nuevas generaciones fueron tomadas muy en cuenta. Sin embargo, las medidas y las políticas afectaron el empleo de los trabajadores yugoslavos en el extranjero y la emigración temporal fue desarrollada en respuesta a los crecientes flujos desde mediados de los años sesenta, aunque sólo en for- 
ma gradual. Así, vimos en los países socialistas europeos una nueva área de política gubernamental que emerge de las realidades y aspiraciones de las políticas de población. Los desarrollos fueron graduales pero no lentos, dentro de una mezcla de políticas ex ante y ex post y aún otras más diversas. La experiencia técnica e intelectual de estos desarrollos fue muy compleja, aunque, puede decirse, señalada por un fuerte ingrediente de interés demográfico. Parece que las políticas están aún en formación, y que nuevos cambios podrán ocurrir en los años venideros.

\section{Desarrollo Reciente de las políticas de fecundidad}

Un estado ideal en el cual una política de población podría operar, esto es, en el cual los objetivos demográficos de la sociedad estuvieran de acuerdo al comportamiento individual, es difícil de imaginar. Si los objetivos estuvieran en completo acuerdo con tal comportamiento, no habría necesidad de tales políticas. La mayoría de las políticas, tienden pues a corregir el curso "indeseable" de los acontecimientos, y a conducirlos hacía un fin "deseable". Esto involucra, desde luego costos y motivaciones, dos temas críticos discutidos en ocasiones, aunque principalmente con respecto a las políticas de los países en desarrollo.

En todos los países socialistas europeos existen efectos externos significativos, que se supone apoyan la existencia de familias numerosas. El gobierno proporciona servicios sociales tales como educación gratuita, servicios de salud, servicios a bajo costo para los niños, asignaciones familiares, vivienda a bajo costo y otras. Sin embargo, la motivación para una familia numerosa es obviamente débil: el comportamiento reproductivo resulta en un nivel de fecundidad menor que el buscado por la política de fecundidad. En términos económicos, el apoyo externo a la familia debe, por tanto, ser considerado insuficiente, o el costo real total de atención infantil demasiado elevado. El elevado estatus de la mujer, las grandes oportunidades de empleo, así como el alto prestigio y la apreciación para el trabajo de la mujer, contribuyen a formar actitudes en que las actividades económicas y sociales compiten con la maternidad. Por otro lado, dentro del clima sociopsicológico dado y en virtud de un control sin restricción de los nacimientos, la decisión para tener un niño más debe darse bajo fuertes presiones. El marco de la política de fecundidad es así muy complejo: requiere de diversas y más refinadas medidas que podrían atraer, de acuerdo a la opinión del planificador, una amplia cooperación del público.

Esta es tal vez una de las razones para dar a la fecundidad el lugar prominente en, al menos, cinco países europeos socialistas. Otra razón puede ser que, debido a la ausencia de migración interna, la fecundidad es la única variable dinámica del crecimiento de la población. Una tercera razón puede ser que las proyecciones demográficas alarmantes indican una disminución de la población en dos países por lo menos, considerando los niveles actuales de mortalidad y fecundidad. 


\section{Objetivos de las politicas de fecundidad}

Existen pocos enunciados explícitos acerca de los objetivos con respecto a la fecundidad. Stancel, el Ministro de Trabajo y Asuntos Sociales de Checoslovaquia, dijo recientemente que era necesario "crear las condiciones necesarias para evitar el decrecimiento de la población después de 1980" para lo cual "se requería que el número de hijos en las familias se incrementara, del 1.9 actual, hasta 2.5 ". ${ }^{21}$ Srb estimó que, considerando el patrón marital, la esterilidad fisiológica y las cifras de mortalidad, un aumento de 2.3 a 2.5 hijos por familia proporcionaría una "reproducción ampliada". Dicho autor estableció que este era un tamaño de familia próximo al considerado como ideal por la mujer checoslova$\mathrm{ca}^{22}$ Klinger afirma que las recomendaciones de políticas, presentadas por algunos expertos en un artículo acerca de políticas de población, corresponden a las propias del gobierno, y que los objetivos de la política húngara es incrementar gradualmente el número de nacimientos" en la medida en que se asegura, al menos, el reemplazo de la población". Otro objetivo es "igualar gradualmente, tanto como sea posible, la estructura de edades".23 En Bulgaria, la Oficina Política del Partido Comunista anunció que la política del partido y del Estado, consistía en ayudar a la mujer a cumplir sus funciones, "de tal manera que se estimule y se valore particularmente su papel de madre". Asimismo, se afirmaba que serían creadas las condiciones óptimas "para elevar la tasa de natalidad y estabilizarla en un nivel que corresponda a nuestro progreso económico y social medio". 24

Las medidas políticas en Checoslovaquia parecen enfatizar la importancia del segundo y tercer hijo; en Hungría y Bulgaria, la del tercero; la política de Rumania parece que aboga por un tercer hijo, pero en algunos casos también por el cuarto hijo. En la República Democrática Alemana, la prima familiar aumenta hasta el quinto niño, con mayor incremento del segundo al tercer hijo. Sin embargo, no todas las medidas conducen a la misma paridez. Se observa que otras consideraciones, aparte de las demográficas, juegan también un papel en el concierto de las medidas individuales. Pero, considerando todas las medidas existentes, parece correcto decir que las políticas de las cinco naciones tienen en común que abogan por un tercer hijo.

No es fácil traducir esto a una tasa demográfica convencional, a menos que otros parámetros demográficos relevantes sean tomados en cuenta.

21 Dr. Matej Štancel, Ministro de Trabajo y Asuntos Sociales de Checoslovaquia, "Populačni politika a jej ulohy" ("La politica de población y su papel". en Checo), Populačni spravy, Núm. 3, 1973, pp. 3-5.

22 Dr. V. Srb, "Reprodukce v manželstvi" (La fecundidad en el matrimonio, en Checo), Populačni spravy. Núm. 4-5, 1972, pp. 21-28.

23 Klinger, véase la nota 25.

24 El destacado papel de la mujer en la construcción de una sociedad socia. lista desarrollada - Decisión del Politburó del Comité Central del Partido Co. inunista Búlgaro, del 6 de marzo de 1973. Sofía Press. 1973, 36 pp. 
No sabemos con precisión lo que significa una política que enfatiza la importancia de un tercer hijo, bajo las variantes condiciones relativas a la situación marital, el estado de la fecundidad, etc. Considerando otro tipo de información, si bien no muy amplia, parece que por lo menos tres países han adoptado políticas dirigidas a elevar la fecundidad ligeramente sobre el nivel de reemplazo, mientras que dos de ellos tienen en mente un crecimiento de población algo más rápido, tal vez semejante al que existía a principio de los años sesenta.

\section{Medidas específicas}

Hay una cierta variedad de medidas promovidas por los gobiernos para alcanzar un estado de reproducción que ellos consideran deseable o satisfactorio. ${ }^{25}$ Tales medidas incluyen legislaciones relacionadas al matrimonio, a la familia y al divorcio; leyes en las que se considera la protección de madres e hijos; regulaciones referentes a servicios de salud; la creación de guarderías, jardines de niños y otras instituciones para el cuidado del niño; programas referentes a la contracepción y el aborto inducido; subsidios familiares; facilidades y garantías para el alumbramiento; vacaciones para la madre antes y después de dar a luz; facilidades pra ayudar a la madre que trabaja y medidas parecidas. Muchas de estas medidas forman parte también de una política de bienestar en los países occidentales, aunque sin hacer referencia a objetivos demográficos. Algunas de ellas han sido desarrolladas originalmente, por ejemplo en los países escandinavos, para impulsar la fecundidad pero, gradualmente, han venido a ser meros componentes del bienestar familiar.

En algunos países, como Hungría, Bulgaria y Checoslovaquia, en los cuales la disponibilidad insuficiente de viviendas se ve como un obstáculo al aumento en la fecundidad, las familias grandes reciben un trato especial al respecto. En otros países como Rumania y Bulgaria, el impuesto sobre la renta es utilizado para estimular a las famiilas con hijos, o para penalizar a aquellos que no tienen hijos o que no son casados. En Checoslovaquia hay una reducción del impuesto solamente para el primer hijo. Los incentivos económicos están también presentes en los programas de subsidios familiares para distinguir, entre los distintos grupos de la población, tales como trabajadores, campesinos, etc. Empréstitos para suministrar y habilitar nuevos hogares son concedidos en Checoslovaquia y son amortizados parcialmente después del nacimiento del primer hijo.

25 Las referencias a esta sección incluyen artículos preparados para un seminario sobre problemas de políticas de población, Versovia, 15-18. marzo de 1972; por F. Khaltbari y otros, acerca de la política de población en la RDA, por M. Latuch acerca de Polonia, por D. I. Valentei y A. J. Kwasha acerca de la URSs; contribuciones para el libro de población compilado por B. Berelson acerca de Hungría por Andras Klinger. Jamisz A. Ziolkowski sobre Polonia y por Petre Maruzan y Ioan M. Copie acerca de Rumania; reportes para una Reunión de Grupo de Trabajo para el estudio de la legislación directa e indirectamente. 
La maternidad es estimulada en todos los países a través de medios diversos, tanto formales como informales. En Rumania, así como en Bulgaria, títulos, distinciones y medallas son otorgadas a madres de familias numerosas. En Hungría, las distinciones y las medallas que comenzaron a otorgarse en 1951, fueron abolidas y reemplazadas en 1957 por primas a las madres de más de seis y con más de once hijos.

Los programas de educación varian de país a país. En Hungría, visitadores sociales y exámenes médicos gratuitos durante el embarazo parecen importantes en la educación de la mujer y en la de las madres futuras. En todos los países, la propaganda en contra del aborto, asi como la información acerca de los contraceptivos, forman parte de los servicios de trabajo social y de salud. En Checoslovaquia hay un programa educacional amplio dirigido a, y hecho para grupos críticos de la sociedad. Existe también una red de servicios de asesoría disponible para las parejas; esos servicios existen en otros países también.

\section{Dirección del cambio}

El cambio en la política se realizó en un doble sentido: por un lado, hubo una expansión y una amplificación de las facilidades y medidas adoptadas tempranamente con base en beneficios sociales y humanitarios; por otro lado, han sido expedidas también nuevas facilidades y servicios. Es obvio que los encargados de llevar a cabo las políticas, así como sus asesores, sabían que lo que importaba en políticas de población no erar las medidas individuales, si no la combinación de medidas, su coordinación y su intensidad. Como lo sugiere un autor húngaro, hay "muchos pequeños detalles de la vida que influyen todavía en el comportamiento de la población".

Las facilidades de salud pública y de servicios médicos, incluyendo asesoría relativa al embarazo, la maternidad y como criar al niño, parecían bastante avanzadas aún antes de las reformas. Sin embargo, algunas innovaciones adicionales han llegado a afectar el cuidado prenatal, la duración de la vida de la madre, el tiempo de asesoramiento relativo al control de la fecundidad y al espaciamiento entre los hijos, etc. En países en los que hay varios programas de seguridad y de salud, algunos servicios se han extendido a las mujeres que no tenían un seguro completo.

Medidas tendientes a contrarrestar las desventajas económicas impuestas por la procreación sobre las familias, fueron ampliadas dentro de recientes reformas a las políticas. Además de las primas familiares que fueron originalmente implantadas con base en la justicia social, los incentivos para estimular el nacimiento de un hijo adicional y la imposición de contribuciones para penalizar a las familias sin hijos, han sido introducidos también en algunos países. Se esperaba que las medidas económicas estimularían la reproducción, más o menos dentro de los lineamientos de la teoría de Landry. ${ }^{26}$ Consecuentemente, nuevas medidas eco-

26 A. Landry, La revolutión démographique, París, 1934. 
nómicas fueron expedidas y experimentadas para aliviar la carga económica de la nupcialidad, de la formación de la familia, la extensión de la familia, etc. En todos los países mencionados, o se aumentó la disponibilidad de guarderías, jardines de niños y de servicios infantiles en general, o se previó una expansión en estos servicios. Debe agregarse que los programas para las generaciones jóvenes son extensivos y amplios en todos los países.

La innovación más importante se refirió al empleo y la elevación del estatus de las mujeres, cuyo progreso ha ido en detrimento de la fecundidad en todos los países socialistas. La idea de reconciliar la maternidad con las funciones sociales se originó a mediados de los años treinta, según Glass, en la Comisión Sueca de Población que estaba "ansiosa por salvaguardar y garantizar el empleo de la mujer casada". ${ }^{27}$ Cambios re. cientes en las políticas, por lo menos en dos países, van mucho más allá dado que proporcionan facilidades alternativas de las cuales la madre puede disfrutar por un largo período, durante el cual son protegidos sus derechos de empleo y pagado parte de su salario. Esto parece ser un desarrollo interesante que merece estudio y atención tanto por los técnicos como por los encargados de diseñar las políticas.

Las medidas educacionales y las medidas dirigidas a la opinión pública han dado alta prioridad a los cambios en las políticas recientes. En algunos países han comenzado programas educacionales sofisticados dirigidos a los distintos grupos claves. La propaganda en favor de una alta fecundidad se ubica dentro de un marco más amplio de "contrucción de una sociedad socialista avanzada". En casi todos los países bajo consideración, las políticas y las medidas han sido adoptadas por los cuerpos administrativos o los grupos políticos importantes. Los funcionarios públicos de más alta jerarquía ocasionalmente emiten enunciados para aumentar el prestigio de una alta fecundidad.

En general, las políticas de población bajo consideración, respetan los derechos individuales de dicidir acerca del número y espaciamiento de los hijos, y no se apoyan en medidas restrictivas. Klinger establece explícitamente que "las medidas administrativas tales como la prohibición del aborto inducido, no conducirán a la estabilización de la población". ${ }^{28}$ Sin embargo, tres países han adoptado políticas restrictivas con respecto al aborto inducido. Los altos precios de los contraceptivos en uno o dos países pueden estar encaminados a producir efectos similares.

Considerándolas como un todo, y guardando las diferencias particulares de cada nación, las reformas en las políticas han afectado la mayoría de los factores que tienden a reducir la fecundidad por debajo del nivel de reemplazo. De acuerdo con estas políticas se proporcionan facilidades a las madres y los hijos, recursos externos para ayudar a la procreación, oportunidades para las generaciones jóvenes, medios para reconciliar la maternidad con las funciones sociales, prestigio y apreciación

27 David Glass, Populations Policies and Movements in Europe, Londres, reimpreso, 490, pp. 1967.

28 Klinger, op. cit. 
por las familias numerosas, programas educacionales y, en algunos casos, medidas restrictivas en contra del aborto. Se espera que todo esto cree una corriente de opinión pública en favor de la procreación, y que estimule a las familias a tener más hijos.

Si a pesar de la combinación de medidas no se obtienen los resultados anunciados, la interrogante que surge es la siguiente: ¿qué grupo de medidas son las que deben hacerse más extensivas? Sin considerar las medidas encauzadas hacia las normas sociales y estándares relativos a la procreación, los factores externos y las medidas adecuadas para armonizar la maternidad y las funciones sociales parecen promisorias debido a su elasticidad. Ambas son versátiles, si bien una y otra requieren de una participación mayor por parte de la sociedad.

Un factor limitante puede ser el elevado gasto en el apoyo externo a las familias y en la compensación a aquellas madres que decidan no trabajar. Otro factor limitante puede ser la oferta de mano de obra, que en los países socialistas depende en alto grado de la participación femenina. Ninguno de estos problemas es tal que sea insoluble, pero requiere de recursos adecuados, de tiempo y de organización. 